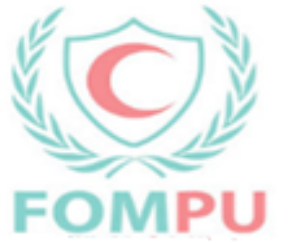

Medicine Updates

Faculty of medicine

July 2020,volume 2 , issue 2 https://muj.journals.ekb.eg

dean@med.psu.edu.eg

vice_dean_postgraduate@med.psu.edu.eg

DOI: $10.21608 / \mathrm{muj} .2020 .29230 .1010$

ISSN : 2682-2741

Submitted: $1 / 5 / 2020$

Accepted : 30/5/2020

Pages:36-52

\title{
SERUM PHOSPHORYLATED NEUROFILAMENT HEAVY CHAIN AS A MARKER OF AXONAL INJURY AND DISEASE SEVERITY IN MULTIPLE SCLEROSIS PATIENTS
}

Adel Saed Abd Elghafar, Professor of Neurology Faculty of medicine, Zagazig University, Egypt

Mahmoud El Sayed El Ebeary, Professor of Neurology Faculty of medicine, Zagazig University, Egypt.

Magdy Abd Elhamed Aidaros, Professor of Neurology Faculty of medicine, Zagazig University, Egypt.

Nagwa mohammed shawky, Professor of Clinical pathology Faculty of medicine, Zagazig University, Egypt.

Doaa abd Elsattar Mohammed, Assistant Lecture of Neurology Faculty of Medicine, Portsaid University, Egypt.

\section{ABSTRACT:}

Axonal damage is the main cause of irreversible neurological complication in multiple sclerosis (MS). Neurofilaments (NFs) are intermediate filaments are present in large amount in axons that responsible for the radial growth of axons and the transmission of electrical impulses. Serum phosphorylated neurofilament-heavy (pNf-H) can be used as a marker to evaluate the presence axonal damage. 
Aim: To evaluate whether serum pNF-H levels are related to disease severity and the disability in MS patients.

Subjects and methods: Study included 35 patients and 35 healthy control. All Subjects were evaluated by history taking, general examination, neurological evaluation and disability scoring by the Expanded Disability Status Score (EDSS), Routine Laboratory Investigations, measuring pNF-H levels and neuroradiological assessment using Magnetic resonance image (MRI) to establish MS diagnosis.

Results: higher levels of pNF-H in MS patients compared to healthy controls. The highest levels of proteins were in the secondary progressive MS and these levels significantly correlated with disease severity measured by EDSS.

Keywords: multiple sclerosis, axonal damage, neurofilament $-\mathrm{H}$.

\section{Introduction:}

Multiple sclerosis is a chronic complex neurodegenerative disease targeting the central nervous system (CNS) and thought to be autoimmune in nature, characterized by the presence of local inflammation, demyelination, gliotic scarring and axonal loss resulted from autoreactive lymphocytes that pass the blood-brain barrier (BBB) and enter the CNS (Huang et al., 2017).

The clinical presentation can include visual disturbance, lassittude, mood disturbance, hemiplegia, paraplegia, parathesia and cognitive affection.To diagnose a chronic neurological disease with severe disability in young adulthood, is almostly devastating and unexpected (Gresle et al., 2011). 
Axonal injury is proven to be the main cause of progressive disability in MS (Kutzelnigg et al., 2005). The most commonly used diagnostic and follow up method for MS are Magnetic resonance imaging especially T1-weighted imaging, T2-weigheted imaging and Gadolinium enhanced. Nowadays markers of axonal injury donot utilized as routine laboratory investigations for diagnosis and evaluating in MS patients (Sahraian and Eshaghi 2010).

An ideal marker of axonal damage should have many charchteristics: it should be present in axons, it must be much enough so can be detected after the significant dilution that occurs after release to fluid compartments as CSF and serum, and it cannot be breaked down by protease before or following release (Anderson et al., 2008).

The phosphorylated subunits of neurofilaments, the major structural protein complexes of axon, meet these criteria. Neurofilament- $\mathrm{H}$ is a major axonal cytoskeletal protein. After axonal injury, they are released into the extracellular compartment and subsequently into the CSF and serum thus analysis of CSF and serum pNF-H levels may provide a way to estimate the extent of axonal injury in patients with MS (Massoud et al., 2018).

\section{Subjects and methods}

This case-control comparative study included 70 subjects, The study was accepted by Institution Review Board at Zagazig University. We selected our patients from internal section and neurology clinic of Zagazig University Hospitals from February 2016 to february 2018. Thirty- five healthy individuals participated as a control group, selected from general population through personal communication. 
(Patients group ) (group 1): 35 patients with clinically definite Multiple Sclerosis. Data about age, sex, age of onest, disease duration, clinical course of the disease, disability type and treatment, divided into, subgroup 1a: Included 8 patients with first demyelinating event (FDE). subgroup 1b: Included 14 patients with relapsing remitting multiple sclerosis (RRMS).subgroup 1c: Included 8 patients Secondary progressive multiple sclerosis (SPMS).subgroup 1d: Included 5 patients primary progressive multiple sclerosis (PPMS). Controls group (Goup2): 35 healthy peoples age and sex matched and they are neurologically normal during physical examination.

All participants should wrote an informed consent and the the research ethical committee of Faculty of Medicine, Zagazig University accepted this study. The work has been carried out in accordance with The Code of Ethics of the World Medical Association (Declaration of Helsinki) for studies involving humans.

\section{Inclusion criteria:}

Definite MS according to the 2010 revised McDonald diagnostic criteria for MS (Polman et al., 2011).

Exclusion criteria: Patients have any other medical diseases (neurological, immunological, cardiac, hepatic, and renal), previous head trauma, pregnancy or transform patients (patients shifted from interferon to fingilimod) were excluded from our study.

\section{Laboratory Invesigations:}

Routine laboratory invesigations : complete blood picture, Liver function test and kidney function tests, Erthrocyte sedimentation rate, Fasting and postprandial blood sugar and lipid profile. 
Special laboratory investigations: Serum phosporylated neurofilament-H chain (pNF-H) levels from both patients and control groups were analyzed using kit (EnCor Biotechnology Inc, Gainesville, Florida, USA) in enzyme-linked immunosorbent assay (ELISA), according to the manufacturer instructions (Boylan et al., 2009).

\section{Statistical analysis}

We used the Statistical Program for Social Science, version 24 (SPSS Inc., Chicago, Illinois, USA) for analysis our data. We used mean \pm SD to express quantitative data. Qualitative data were expressed as frequency and percentage. The following tests were done ,Mann-Whitney $U$ test (MWT). We correlated our data by Pearson's correlation coefficient (r): Probability ( $\mathrm{P}$ value): (a) considered significant if $\mathrm{P}$ value less than 0.05 was. (b) considered as highly significant if $\mathrm{P}$ value less than 0.001.(c) insignificant when $P$ value greater than 0.05 (Coolidge, 2012).

\section{Results:}

This case control comparative study included thirty five MS patients , their age ranged between 19 to 61 years with $\mathrm{M} \pm \mathrm{SD}=38.7 \pm 12.5$ , $37.1 \%$ ( 13 patients) were males, $61.9 \%$ (22 patients ) were females (table 1).

There was highly statistically significant difference in the serum pNF-H levels between MS patients and controls (pNF-H levels in the patients is significant higher than control group) (table 2).

There was highly Statistically significant difference in the serum levels of pNF-H between MS subtypes the highest levels in the secondary 
progressive group ( Table3). There was direct correlation between serum pNF-H levels, disease duration, EDSS (table 4, 5).

There was no significant difference in the serum levels of pNF-H as regard the presence and absence of black hole sign in T1 figure(1). There was significant difference in serum pNF-H between treated and nontreated patients with the lowest levels in patients treated with fingolimod (figure 2).

\section{Discussion:}

Our study included 35 patients, 8 patients with FDE (22.8\%), 14 patients with RRMS (40\%), 8 patients with SPMS (22.8\%) and 5 patients with PPMS (14.4\%) and 35 healthy controls.

Our study included females more than males with 23 females and 12 male with a ratio 1.9:1. Our results are as the same results of previous studies about MS which have shown high prevalence of MS in young adults and females (Mc Combe and Greer 2013). Also in agreement with an Egyptian multiple sclerosis multicenter registry study that confirmed female dominance in MS (Sherif et al., 2017) with female:male ratios of 2.14:1.0. In a previous study, in the Arab countries, the female:male ratio varies widely in the Arab countries, eg, from 0.8 in Oman to 4.3 in Saudi Arabia, and the overall estimate of female proportion in MS patients was $0.67(0.65-0.69)$ (Heydarpour et al., 2015).

The immunoglobulins levels are higher in women than men and during the peri-ovulatory period there is a decreased cell-mediated immunity and natural killer (NK) cell activity. Also estrogen and progesterone can modify interleukin-1 (IL-1) production in vitro. The lymphoid cells or the thymic matrix contain cytoplasmic receptors for 
estrogens and androgens, this illustrate why these sex hormones can modify the function of these organs and to interact with the immune system and regulate it (Duquette et al., 1992).

The serum pNF-H was shown to be significantly higher in our MS patients than controls $(\mathrm{p}<0.00)$. Neurofilaments, a major cytoskeletal componant of neuronal cells, can be released into the cerebrospinal fluid and serum in cases of axonal injury in different neurological diseases such as multiple sclerosis. This finding is in line with another study found that the level of pNF-H was higher in all MS subtypes patients than control (Petzold, 2005). This result also in agreement with that of Kuhle et al (2011) who stated that pNF-H levels were found to be higher in MS patients in all disease stages compared to control. Also with Massoud et al (2018) who found there was a highly statistically significant difference in the levels of pNF-H in patients with MS when compared with control group.

There is highly significant difference in the serum levels of pNF-H between MS subtypes in our study ( $\mathrm{sig}=<0.001$ ), the highest levels of neurofilament are in the secondary progressive group. This finding indicate that the patients with SPMS have more CNS axonal injury caused by inflammatory mechanisms. Detection of neurofilament and its breakdown peptides within the serum can predict definitive axonal damage and disease progression. These results are in line with the work of Gresle et al (2014) who found that Patients with SPMS were had a higher level of pNF-H more than RRMS and FDE patients. They measured serum pNF-Hby ELISA in case with RRMS, SPMS, PPMS and FDE they found that a higher level of pNF-H was in 9\% of relapsing 
remitting and first demyelinating and $38.5 \%$ of secondary progressive multiple sclerosis cases (Gresle et al., 2014).

Higher levels of neurofilament heavy chain in patients with more progressive disease would imply a continuous neurofilament release and thus reflect a severe irreversible neurodegenerative process in these patients (Gunnarsson et al.,2011).

Also Petzold (2005) found that patients of all subtype of multiple sclerosis have higher pNF-H with 1.5 fold higher for progressive multiple sclerosis relative to relapsing remitting multiple sclerosis cases. So that the ongoing neurodegenerative activity of multiple sclerosis patients can be measured by phosphorylated neurofilament heavy chain level which would make this protein a potential candidate for us as a surrogate marker for assessment of treatment aimed at reducing axonal damage.

This is in line with previous follow up study on MS patients, they were followed up fifteen years. The patients with multiple sclerosis were classified relapsing remitting MS and progressive disease, as significant increase in neurofilament heavy chain from base line to follow up, was revealed in a high percentage of patients with primary progressive multiple sclerosis / secondary progressive when compared to patients relapsing remitting multiple sclerosis. The marked increase of neurofilament heavy chain levels implies that loss of neurological function is a direct result of axonal injury and axonal damage is a gradual cumulative process during the course of the disease (Petzold, 2015).

Massoud et al (2018) also assess pNF-H for all their patients , they found that the mean level of pNF-H in RRMS group was 93.5 \pm 245.6 , with a range of 6.2-1130.5 and the mean level of PNF-H in SPMS group 
was $895.8 \pm 1003.7$ with a range of $46.1-4000$. There was a highly statistically significant difference between the two groups and the heighest level in patient with SPMS.

Our finding of a positive correlation between patient disability measured by EDSS and pNF-H protein levels is in line with previous study found that p-NFH levels correlated with EDSS scores in patients with CIS and RRMS and during relapse, the correlation was most prominent in RRMS during relapse (Kuhle et al., 2011). Also another study found a positive correlation between patient disability and pNF-H protein levels (Fialova et al., 2013). Massouad et al (2018) also worked on the relation between pNF-H and expanded disability status scale, they showed that the level of PNF-H significantly increased with the progression of MS as measured by expanded disability status scale this mean that we can depend on pNF-H for assessing disease severity.

In our study there was significant difference in serum pNF-H between untreated patient (did not take disease modifying treatment yet) and treated patients on disease modifying treatment (either fingilomod or interferon), with the highest levels of pNF-H are in untreated group and the lowest levels of pNF-H are in patients on fingilomod.

The first oral MS disease-modifying therapy was Fingolimod, and it is the first drug to be targetting sphingosine-1-phosphate receptors, compared to all previously approved MS therapies it has a fundamentally different and validated molecular target. It is phosphorylated to its active metabolite, Fingolimod -P. Fingolimod-P Fingolimod after passing the blood brain barrier (BBB) into the CNS then bind with S1P receptors that present on neuron, astrocyte, microglia and oligodendrocytes, also on 
vascular endothelial cells of BBB. Fingolimod has a direct CNS effects so can decrease demyelination and promote remyelination. Results from phase 3 trials of fingolimod indicate that the preservation of neural cells and axons observed may be due to its efficacy on brain atrophy outcomes in MS patients. There is increase the benefits to be used in non-relapsing forms of MS because of its ability for direct CNS preservation effects (Groves et al., 2013).

These results are in line with those reported by a previous study on their work on neurofilament light chain they stated that neurofilament is sensitive to treatment. Firstly neurofilament assessment, then after start of study treatment at six months, they found that blood neurofilament concentrations in placebo and IFN- $\beta-1 \mathrm{a}$ were significantly higher compared in the fingolimod group and neurofilaments levels in patients treated with fingolimod were approaching to levels in healthy control (Kuhle et al., 2019).

AS regard as black hole sign, we reported no significance difference in the level of pNF-H and presence or absence of black hole sign on T1. This is in contrast to Massoud et al (2018) who found a significant correlation between black holes and pNF-H level, which was significantly increased in patient with SPMS. 'Black holes' nonenhancing lesions that are present on T1-weighted sequences. We differ from them in having only three patients with positive black hole sign but they had twenty three patients. The formation and conversion rate of acute gadoliniumenhancing lesions to chronic BHs is reduced by Both older and newergeneration disease modifying therapy so its incidence decrease (Marziniak and Meuth 2014). 
Hemond and Bakshi stated that $40 \%-60 \%$ of the acute T1-hypointensities associated with gadolinium-enhancing lesions within six to twelve months will change to $\mathrm{T} 1$ isointense tissue and chronic black holes (BHs) are the remaining lesions,typically present in the supratentorial areas, these mean severe irreversible demyelination and axonal loss. The greater the loss of axonal density and matrix destruction revealed by histological correlation lead to more profound $\mathrm{T} 1$ hypointensity in the persistent BHs. Longer duration of enhancement and larger lesions will have higher risk for conversion from acute to chronic BHs, SPMS tends to show a higher BHs overload compared to relapsing MS ( Hemond and Bakshi 2018).

\section{Referances:}

Anderson KJ, Scheff SW, Miller KM, Roberts KN, Lesley K and etal (2008): The Phosphorylated Axonal Formof the Neurofilament Subunit NF-H (pNF-H) as a Blood Biomarker of Traumatic Brain Injury. Journal of Neurotrauma; 25:1079-1085.

\section{Boylan K, Yang C, Crook J, Overstreet K, Heckman M, and etal} (2009): Immunoreactivity of the phosphorylated axonal neurofilament $\mathrm{H}$ subunit (pNF-H) in blood of ALS model rodents and ALS patients: evaluation of blood pNF-H as a potential ALS biomarker. J Neurochem; 111:1182-1191.

Coolidge F (2012): Statistics a gentle introduction 3rd edition.SAGE Publication.1-38.

Duquette P, Pleines J, Girard M, Chares L, Senecal-Quevillon M and etal (1992): The Increased Susceptibility of Women to Multiple Sclerosis. Can. J. Neurol. Sci. 1992; 19: 466-471. 
Fialova LI, Bartos A, Svarcova J, Zimova D, KotoucovaJ and etal (2013): Serum and cerebrospinal fluid light neurofilaments and antibodies against them in clinically isolated syndrome and multiple sclerosis. JNeuroimmunol;262:113-20.

Gresle MM, Butzkueven H, Shaw G (2011): Neurofilament proteins as body fluid biomarkers of neurodegeneration in multiple sclerosis.Multiple sclerosis international;2011:1-7.

Gresle M, Liu Y, Dagley L, Haartsen J and Pearson F (2014):Serum phosphorylated neurofilament- heavy chain levels in multiple sclerosis patients. J Neurol Neurosurg Psychiatry;85:1209-1213.

Groves A, Kihara Y, Chun J (2013): direct CNS effects of sphingosine1-phosphate (S1P) receptor modulation and implication in multiple sclerosis therapy.J Neurol Sci;328:9-18.

Gunnarsson M, Axelsson M, Sundstro P, Dahle C, Vrethem M and etal (2011): Axonal Damage in Relapsing Multiple Sclerosis is Markedly Reduced by Natalizumab. ANN NEUROL;69:83-89.

Hemond CC and Bakshi R (2018): Magnetic Resonance Imaging in Multiple sclerosis. Cold spring Harb Perspect Med;8: 69-81.

Heydarpour P, Khoshkish S, Abtahi S, Moradi LM and Sahraian MA(2015): Multiple sclerosis epidemiology in Middle East and North Africa: a systematic review and meta-analysis. Neuroepidemiology;44:232-44

Huang W, Chen W, Zhang X (2017): Multiple sclerosis: Pathology, diagnosis and treatments. Experimental and theraputic medicine; 13: 3163-3166. 
Kuhle J, Kropshofer H, Dieter A, Kundu U, Meinert R, and etal (2019): Blood neurofilament light chain as a biomarker of MS disease activity and treatment response. Neurology;92: 1-9.

Kuhle J, Leppert D, Petzold A, Regenitter A, Schindler C, and etal (2011): Neurofilament heavy chain in CSF correlates with relapse and disability in multiple sclerosis. J Neuroimmunol ;220:114-119.

Kutzelnigg A, Lucchinetti CF, Stadelmann C, Bruck W, Rauschkan H and etal (2005): Cortical demyelination and diffuse white matter injury in multiple sclerosis. Brain; 128: 2705-2712.

Marziniak M and Meuth S(2014): Current perspectives on interferon $\beta$ $1 \mathrm{~b}$ for the treatment of multiple sclerosis. Adv Ther 31: 915-931

Massoud HM, Saif EM, Khattabb SS, Saed GA (2018): Neurofilament as a bio marker of axonal loss in multiple sclerosis. Sci J Al-Azhar Med Fac, Girl;1:63-68.

Mc Combe $\mathbf{P}$ and Greer $\mathbf{J}$ (2013): Female reproductive issues in multiple sclerosis. Mult Scler. J;19:392-402.

Petzold A (2005): Neurofilament phosphoforms: surrogate markers for axonal injury, degeneration and loss. Journal of the Neurological Sciences;233:183-198.

Petzold A (2015): The prognostic value of CSF neurofilaments in multiple at 15 years follow up. Neurol Neurosurgery Psychiatry;68:13881390

Polman CH, Reingold SC, Banwell B, Clanet M, Cohen JA and etal (2011): Diagnostic criteria for multiple sclerosis: 2010 revisions to the mcdonald criteria. Ann. Neurol. ; 69:292-302. 
Sahraian MA and Eshaghi A (2010): Role of MRI in diagnosis and treatment of multiple sclerosis. Clinical Neurology and Neurosurgery;112:609-615.

Sherif H, Maged A, Nevin M , Alaa N, Ahmed A and etal (2017): Characteristics and predictors of progression in an Egyptian multiple sclerosis cohort: a multicenter registry study Neuropsychiatr Dis Treat; (13): 1895-903.

\author{
tabels
}

Table (1): Demographic data of our patients.

\begin{tabular}{|c|c|c|}
\hline & & Group \\
\hline & & MS \\
\hline & & $\mathrm{N}=35$ \\
\hline \multirow{2}{*}{ Gender } & Male & $13(37.1 \%)$ \\
\hline & Female & $22(62.8 \%)$ \\
\hline \multicolumn{2}{|c|}{ Age } & $38.7 \pm 12.5$ \\
\hline
\end{tabular}

MS= multiple sclrerosis

This table shows that thirty five MS patients participated in our study, their age range from 19 to 61 with $\mathrm{M} \pm \mathrm{SD}=38.7 \pm 12.5$. $37.5 \%$ (13 patients) are males. $62.5 \%$ (22 patients) are females. 
Table (2): Comparsion of Serum phosphorylated -heavy neurofilament levels between patient group and control group.

\begin{tabular}{|c|c|c|c|c|c|c|c|c|c|c|c|}
\hline \multirow{2}{*}{ Group } & \multicolumn{3}{|c|}{$M S$} & \multicolumn{3}{|c|}{ CONTROL } & \multicolumn{3}{|c|}{ Total } & \multirow{2}{*}{$M W T$} & \multirow{2}{*}{$P$} \\
\hline & $\mathrm{N}$ & Median & Range & $\mathrm{N}$ & Median & Range & $\mathrm{N}$ & Median & Range & & \\
\hline $\begin{array}{c}p N F- \\
H\end{array}$ & 35 & 0.887 & $\begin{array}{l}0.401- \\
12.285\end{array}$ & 35 & 0.575 & 0.261-0.669 & 80 & 0.629 & $\begin{array}{l}0.261- \\
12.285\end{array}$ & 6.9 & $<0.001$ \\
\hline
\end{tabular}

(MWT) Mann-Whitney U test, MS= multiple sclerosis

pNF-H $=$ phosphorylated - neurofilament heavy chain

Table (3): Comparison of serum phosphorylated - neurofilament heavy chain levels according to multiple sclerosis subtype.

\begin{tabular}{|c|c|c|c|c|c|c|}
\hline & FDE & PP & RR & SP & KWT & Sig. \\
\hline pNF-H & $0.8(0.4-1)$ & $0.8(0.5-1)$ & $0.9(0.5-1.6)$ & $5.8(1.1-12.3)$ & 11.3 & $<0.001$ \\
\hline
\end{tabular}

(KWT)Kruskal-Wallis test

$\mathrm{PP}=$ primary progressive MS

$\mathrm{SP}=$ secondary progressive $\mathrm{MS}$

pNF-H $=$ phosphorylated - neurofilament heavy chain
$\mathrm{FDE}=$ first demyelinating event

$\mathrm{RR}=$ relapsing remitting $\mathrm{MS}$ 
Table (4) Correlations between serum phosphorylated neurofilament heavy chain level and Expanded disability status scale.

\begin{tabular}{|c|c|c|}
\hline \multirow{2}{*}{ MS } & \multicolumn{2}{|c|}{ pNF-H } \\
\cline { 2 - 3 } & $\mathrm{r}$ & $\mathrm{P}$ \\
\hline \multirow{2}{*}{ EDSS } & 0.531 & $<0.001$ \\
\hline
\end{tabular}

$r=$ Correlation Coefficient $\quad$ EDSS= expanded disability status scale pNF-H= phosphorylated - neurofilament heavy chain

Table (5): Correlations between serum phosphorylated - neurofilament heavy chain level and the disease duration

\begin{tabular}{|c|c|c|}
\hline \multirow{2}{*}{ MS } & \multicolumn{2}{|c|}{$p N F-H$} \\
\cline { 2 - 3 } & $r$ & $P$ \\
\hline$D D$ & 0.657 & $<0.001$ \\
\hline
\end{tabular}

$r=$ Correlation Coefficient EDSS= expanded disability status scale

$D D=$ disease duration 
Figures

Figure (1): Comparsion of serum phosphorylated - neurofilament heavy chain levels in multiple sclerosis patients as regard black hole sign in T1 MRI

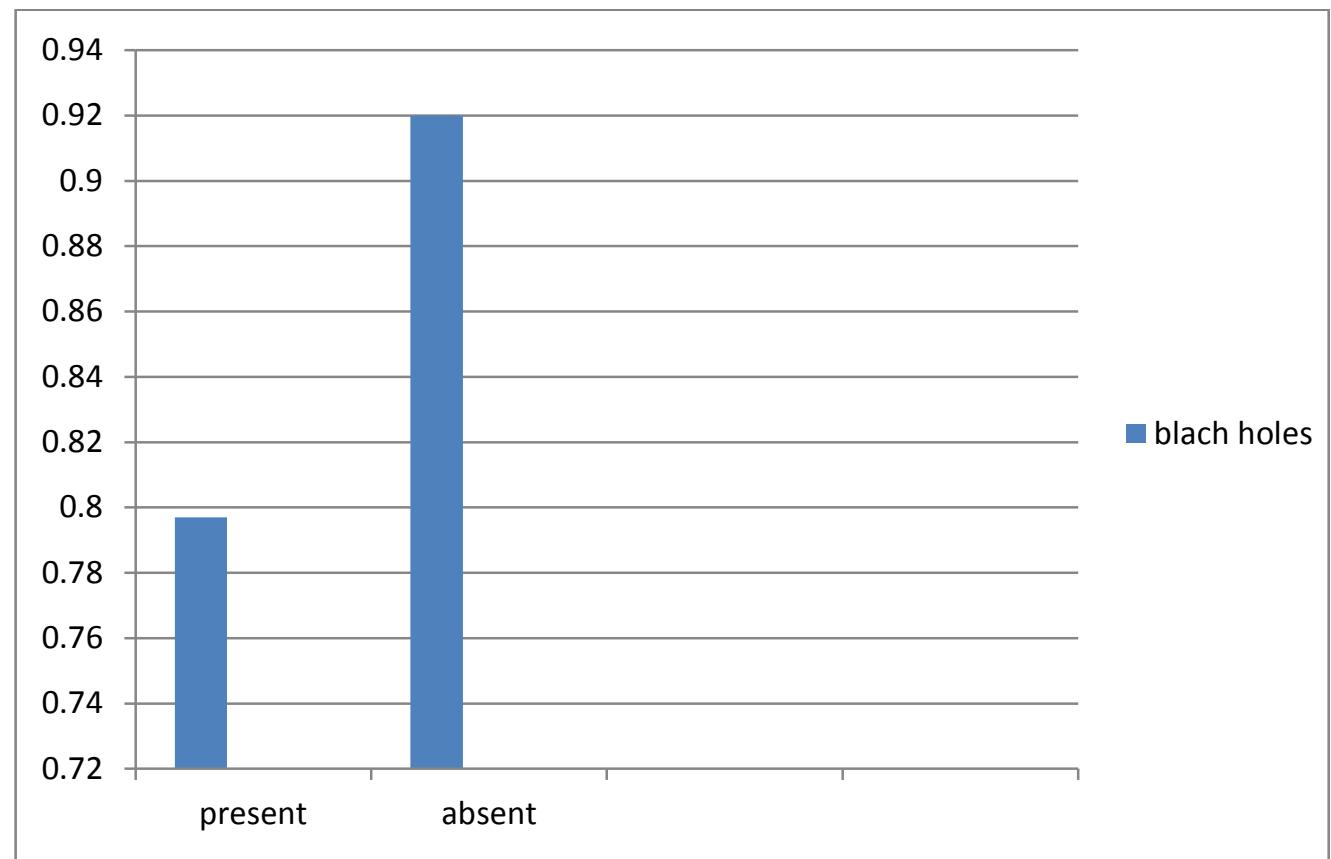

Figure (2): Comparison of serum phosphorylated-neurofilament heavy chain levels in multiple sclerosis group as regard therapy

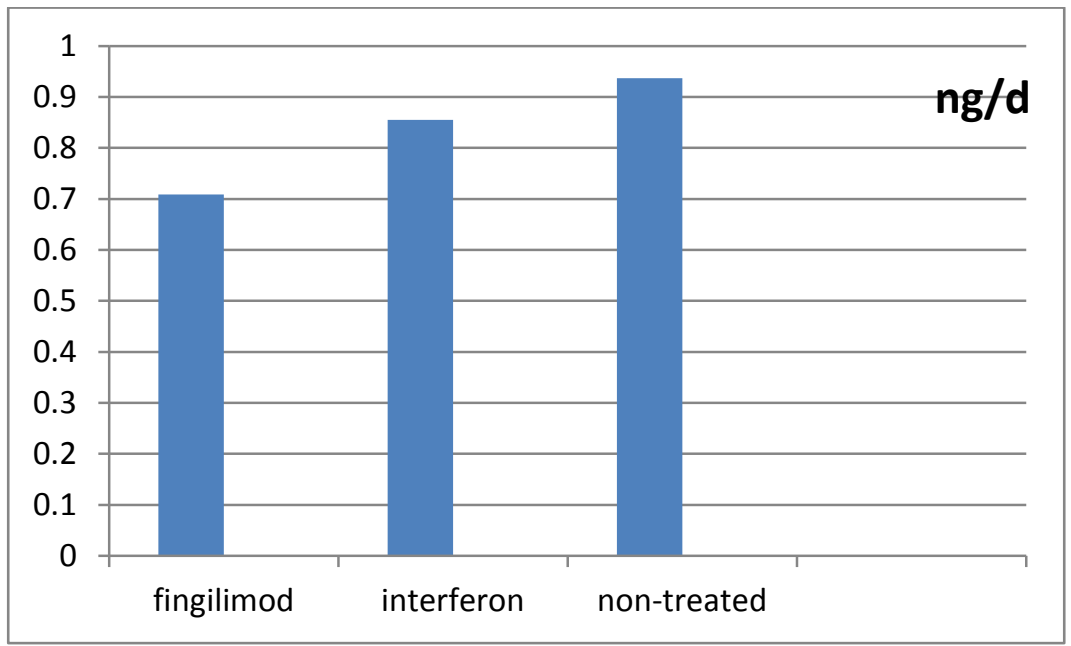

